\title{
uvby photometry of the magnetic CP stars 20 Eridani, HR 1297, 12 Canis Majoris, and HD 134214
}

\author{
Saul J. Adelman \\ Department of Physics, The Citadel, 171 Moultrie Street, Charleston, SC 29409, U.S.A.
}

Received May 2; accepted June 15, 2000

\begin{abstract}
Differential Strömgren uvby observations from the Four College Automated Photoelectric Telescope (FCAPT) are presented for the $\mathrm{mCP}$ stars 20 Eri, HR 1297, 12 CMa, and HD 134214. Adelman \& Boyce found suggestions that the light curves of 20 Eri change between observing seasons. Observations for two additional seasons confirm this behavior and lead to an improved period of 1.928890 days. For HR 1297 a new period of 15.7490 days was derived. The uvby light curves now appear to be the same for different observing seasons. $12 \mathrm{CMa}$ is found to be a photometric variable with the 2.18010 day period determined from magnetic measurements by Bohlender et al. Observations of the rapidly oscillating Ap star HD 134214 confirm the lack of photometric variability which is not associated with the rapid oscillations.
\end{abstract}

Key words: stars: chemically peculiar — stars: individual: 20 Eri — stars: individual: HR 1297 — stars: individual: $12 \mathrm{CMa}$ - stars: individual: HD 134214

\section{Introduction}

This paper discusses single-channel differential Strömgren uvby observations of the magnetic Chemically Peculiar (mCP) stars 20 Eri, HR 1297, 12 CMa, and HD 134214 obtained with the Four College Automated Photoelectric Telescope (FCAPT). As both 20 Eri and HR 1297 were found to possibly exhibit changes in the shapes of their light curves by Adelman \& Boyce (1995) and by Adelman \& Brunhouse (1998), respectively, new sets of observations were made to further investigate this possibility for these stars. $12 \mathrm{CMa}$ is a mCP star whose period was found from

Send offprint requests to: Saul J. Adelman

* Tables 2, 3, 4 and 5 are only available in electronic form at the CDS via anonymous $\mathrm{ftp}$ to cdsarc.u-strasbg.fr (130.79.128.5) or via http://cdsweb.ustrasbg.fr/Abstract.html magnetic field measures, but lacked photometry around its cycle of variability while HD 134214 is a rapidly oscillating Ap star.

The emergent energy distributions, photospheric abundances, and magnetic field strengths of mCP stars depend upon photospheric position. A distant observer observes photometric, spectrum, and/or magnetic variability due to rotation when the geometry is favorable and the surface ranges in abundance and in magnetic field strength are sufficient since the magnetic and rotational axes often make a large angle with each other. Diffusion and gravitational settling and other hydrodynamical processes in radiative atmospheres and envelopes which have strong magnetic fields are thought to produce their anomalous photospheric abundances which depend both on the local magnetic field strength and the time since the star was on the ZAMS (Michaud \& Proffitt 1993 and references therein). Photometric studies using data from the FCAPT have both improved periods and better defined the shapes of their light curves (see, e.g. Adelman et al. 1999). If spectra of these stars are obtained as a function of rotational phase, the distribution of surface abundances can be derived for stars which show modest to moderate rotation.

Since September 1996 the FCAPT has been at Washington Camp, AZ, after previously operating from Mt. Hopkins, AZ. It first measures the dark count for each group of variable, check, and comparison star. In each filter it then observes the sky $-c h-c-v-c-v-c-v-c-c h-$ sky where sky is a reading of the sky, $c h$ that of the check star, $c$ that of the comparison star, and $v$ that of the variable star. The photometer uses a thermoelectrically cooled GaAs Hammatsu photomultiplier (Genet et al. 1987). Table 1 contains group information (Hoffleit 1982, SIMBAD database). In the magnitudes discussed in this paper corrections were not made for neutral density filter differences among the stars of each group. The comparison and check stars were chosen from supposedly non-variable stars near the variable on the sky that had similar $V$ magnitudes and 
Table 1. Photometric groups

\begin{tabular}{rllcl}
\hline HD Number & Star Name & Type & $V$ & Spectral Type \\
\hline 22470 & 20 Eri & $v$ & 5.23 & B9p \\
23055 & HR 1128 & $c$ & 6.59 & A3V \\
23754 & $\tau^{6}$ Eri & $c h$ & 4.23 & F3III \\
& & & & \\
26571 & HR 1297 & $v$ & 6.12 & B9IIIp:Si: \\
27176 & 51 Tau & $c$ & 5.65 & F0V \\
24740 & 32 Tau & $c h$ & 5.63 & F2IV \\
& & & & \\
49333 & 12 CMa & $v$ & 6.08 & B7IIIn \\
47827 & HR 2452 & $c$ & 6.05 & A0 \\
46933 & $\xi^{2}$ CMa & $c h$ & 4.54 & A0V \\
& & & & \\
134214 & BD $13^{\circ} 4081$ & $v$ & 7.7 & Ap \\
132230 & 17 Lib & $c$ & 6.60 & A1V \\
137052 & $\epsilon$ Lib & $c h$ & 4.94 & F5IV \\
\hline
\end{tabular}

$B-V$ colors. Later Adelman et al. (1998) checked their stability using Hipparcos photometry (ESA 1997). The Scargle periodogram (Scargle 1982; Horne \& Baliunas 1986) and the clean algorithm (Roberts et al. 1987) are used to help find the rotational periods.

\section{20 Eri}

Adelman \& Boyce (1995) used 78 FCAPT uvby observations to improve the ephemeris of Renson \& Manfroid (1981) for the mCP star 20 Eri (HR 1100, HD 22470) whose $u, v, b$, and $y$ magnitudes vary in phase and found

HJD (light minimum) $=2443485.50+1.92893 E$.

Small differences in the light curves including their shapes and their amplitudes suggested that 20 Eri might be showing a second period perhaps due to precession. Hence in the 1997-98 and 1998-99 observing seasons 44 and 86 new FCAPT observations, respectively, were obtained (Table 2). Plotting the new observations using the previously adopted ephemeris shows a phase shift. As the light curves are shifted toward smaller phase values, the period should be increased to $1.928890 \pm 0.00005$ days while retaining the zero phase value. To bring the previous $u, v$, $b$, and $y$ FCAPT values into agreement with those reported in this paper for 1998-99, -0.02, 0.05, 0.033, and $0.022 \mathrm{mag}$, respectively, need to be added. These differential shifts most likely result from differential extinction errors exposed by the relocation of the telescope in 1996 . They are worse for 20 Eri than most other stars due to its Southerly declination. No shifts were seen between 199798 and 1998-99 values for $u$ and $v$, but the 1997-98 $b$ and $y$ required 0.015 and $0.012 \mathrm{mag}$, respectively, to added for agreement. To make the values of Renson \& Manfroid (1981) also agree $0.89,1.18,1.213$, and 1.25 mag must be added to the published $u, v, b$, and $y$ values, respectively. Both sets of observations used HR 1128 as a comparison star, but they used $\tau^{5}$ Eri instead of $\tau^{6}$ Eri as the other constant star.

If a $\mathrm{mCP}$ star shows a second period due to precession of the rotational axis, then its various spots and back- ground regions will transit across the visible surface with periodically varying distances from the center of the visible disk. This will result in changes in the amplitudes of all maxima and minima as well as in the phases of the rising and falling branches of the light curves. When the precessional period is known, one can remove the effects of the precessional period on the rotational period. Until then, attempts to find the best period by matching the primary maximum for 20 Eri, may emphasize the differences at other parts of the rotational cycle. The rotational period can be found when light curves from several consecutive years are the same as other light curves from a similar set of consecutive years. This has not happened yet for 20 Eri and may be the result of not obtaining a sufficient number of significantly well determined light curves in different observing seasons.

Figure 1 shows the $u, v, b$, and $y$ photometry of this paper for 1997-98 (as solid circles) and for 1998-99 (as open squares), of Renson \& Manfroid (1981) (as + signs), and of Adelman \& Boyce (1995) for 1993-94 (as open upward pointing triangles). Figure 2 shows these $u$ values along with those of Adelman \& Boyce (1995) for 1990-91 (as solid squares), for 1991-92 (as solid downward pointing triangles), for 1992-93 (as x's), and for 1994-95 (as open circles). These additional values were not included in Fig. 1 both as these data sets were not as complete and to simplify the graphs.

In Fig. 1, the $u$ values for 1998-99 tend to be lower and scatter more at primary maximum than the other data. The 1997-98 values tend to show a less deep primary minimum than the Renson \& Manfroid (1981) and Adelman \& Boyce (1995) 1993-94 values than do the 1998-99 values. In Fig. 2, the 1990-91 and 1991-92 values show an upward displacement of order 0.01 magnitude near phase 0.7 while the 1992-93 and 1994-95 values are shifted downward here. These all indicate that the $u$ light curve is changing and is consistent with what is expected for precession. The $v$ values for 1997-98 and for 1998-99 differ mostly at primary minimum. The differences between sets of values is the least for $b$. For $y$ it is the Renson \& Manfroid (1981) values which show a deeper minimum that those of 199899 which in turn a deeper minimum than do the 1997-98 values.

These results indicating subtle, yet observable changes in the shapes of the light curve mean further observations of 20 Eri are warranted. It is desirable to obtain light curves with of order 50 to 100 values to well define them. If the pattern of light curve changes begins to repeat, then the period is determined.

\section{HR 1297}

Adelman \& Brunhouse (1998) used 79 uvby observations from the FCAPT and the $V$ values of Winzer (1974) to find a period of 1.06457 days which was similar to that of Winzer. As they found small apparent differences 
20 Eri
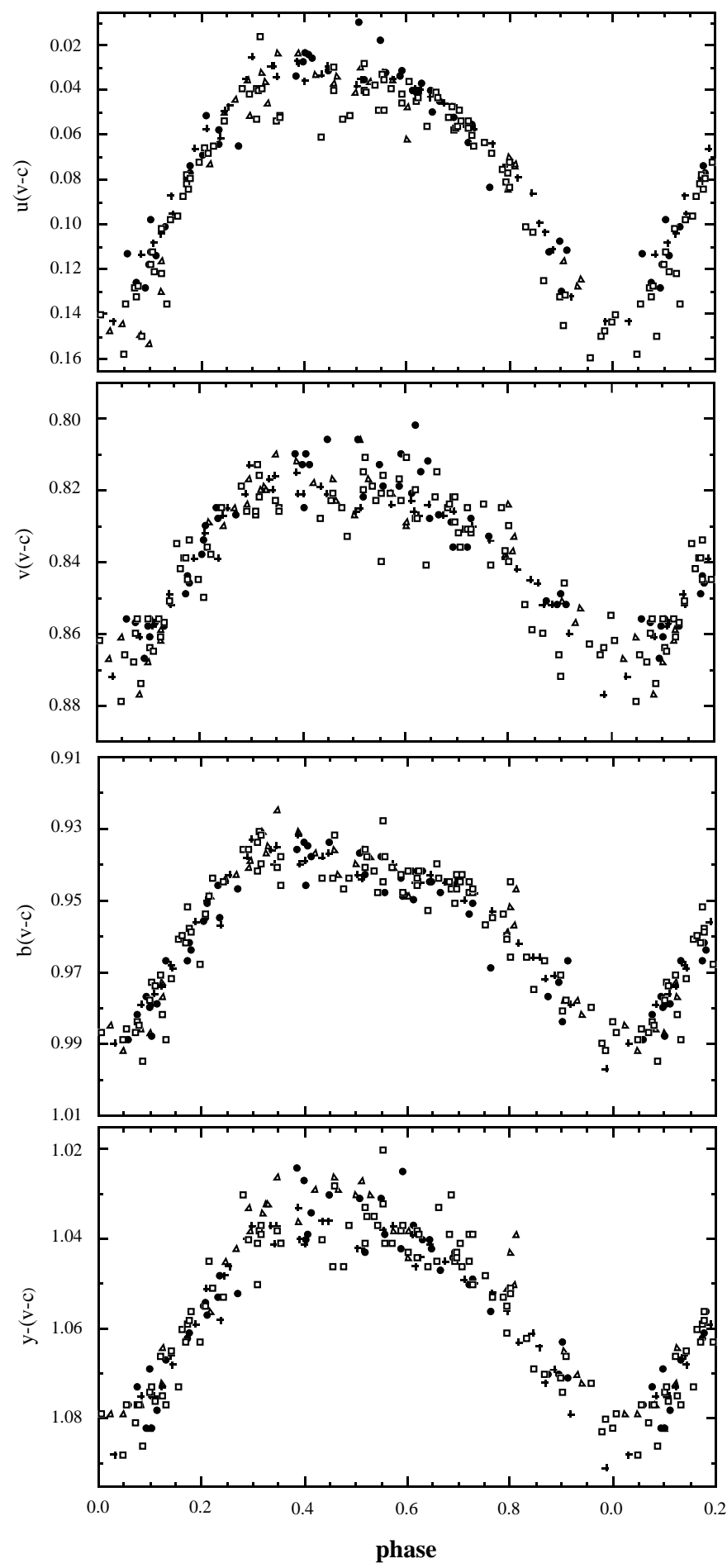

Fig. 1. Differential FCAPT uvby photometry of 20 Eri plotted with the ephemeris HJD (light minimum) $=2443485.50$ $+1.92889 \mathrm{E}$. The 1998-99 FCAPT values are shown as open squares, the 1997-98 FCAPT values as solid circles, Renson \& Manfroid (1981)'s values as + signs, and the FCAPT 1993-94 values from Adelman \& Boyce (1995) as solid triangles
20 Eri

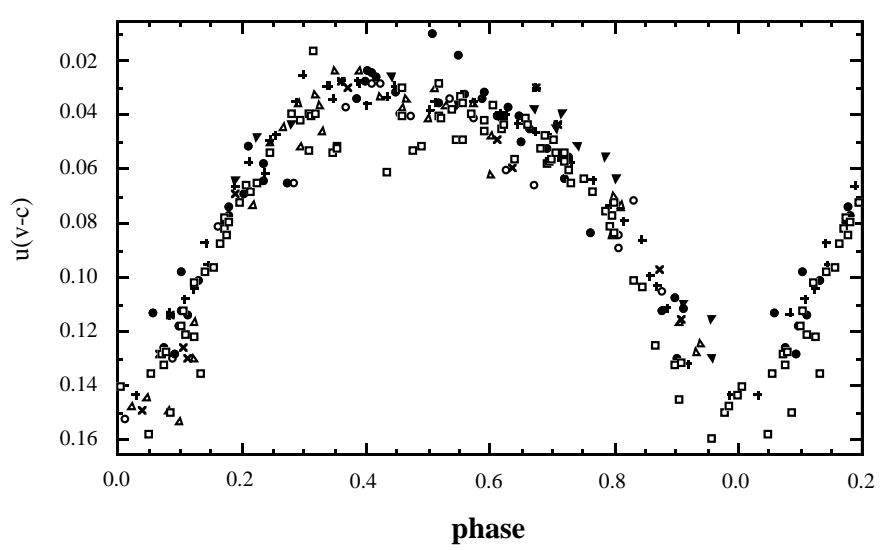

Fig. 2. Differential FCAPT $u$ photometry of 20 Eri using the ephemeris HJD (light minimum) $=2443485.50+1.92889 E$. The symbols are the same as those of Fig. 1 with the addition of FCAPT values from Adelman \& Boyce (1995): solid squares are 1990-91 values, solid triangles with downward pointing apexes are 1991-92 values, x's are 1992-93 values, and open circles are 1994-95 values

between light curves obtained in the 1995-96, the 1996-97, and the 1997-98 observing seasons, HR 1297 was observed again during the 1998-99 observing season at which time 117 good uvby observations were obtained to better define the light curves. When the Scargle periodogram was used with the new data for $u$, there were two significant peaks one corresponding to the previously determined period and one to its alias near 15.723 days. In the new data set sometimes two or more values were obtained on the same night. As these pairs of values showed small differences and as Catanzaro et al. (1999) indicated a $v \sin i$ value of $29 \mathrm{~km} \mathrm{~s}^{-1}$, the longer period was preferred. To bring the $V$ data of Winzer (1974) into phase agreement with FCAPT $y$ values, the following ephemeris was found

$\operatorname{HJD}\left(y_{\max }\right)=2441247.475 \pm 0.005+(15.7490 \pm 0.0002) E$.

With this period, the FCAPT values from different observing seasons appear to form the same light curve contrary to the previous observations (Fig. 3). As for year 9 a $2.5 \mathrm{mag}$ neutral density filter was removed, to bring the $u, v, b$, and $y 1999$ values into agreement with previous FCAPT values $-2.435,-2.452,-2.380$, and $-2.403 \mathrm{mag}$, respectively, were added. The light curves are in phase with suggestions that the primary minimum consists of two minima in some light curves. For $u$, there is one near phase 0.30 and the other near phase 0.6. The minima for $v$ and $b$ are less flat bottomed that for $u$ and more asymmetric. For $y$ the minimum is again flat bottomed but it is smaller in phase extent than for $u$. The amplitudes for $u, v, b$, and $y$ are, $0.045,0.035,0.035$, and $0.035 \mathrm{mag}$, respectively. The differences in the shapes of these light curves suggests a photosphere with somewhat variable properties. 
HR 1297
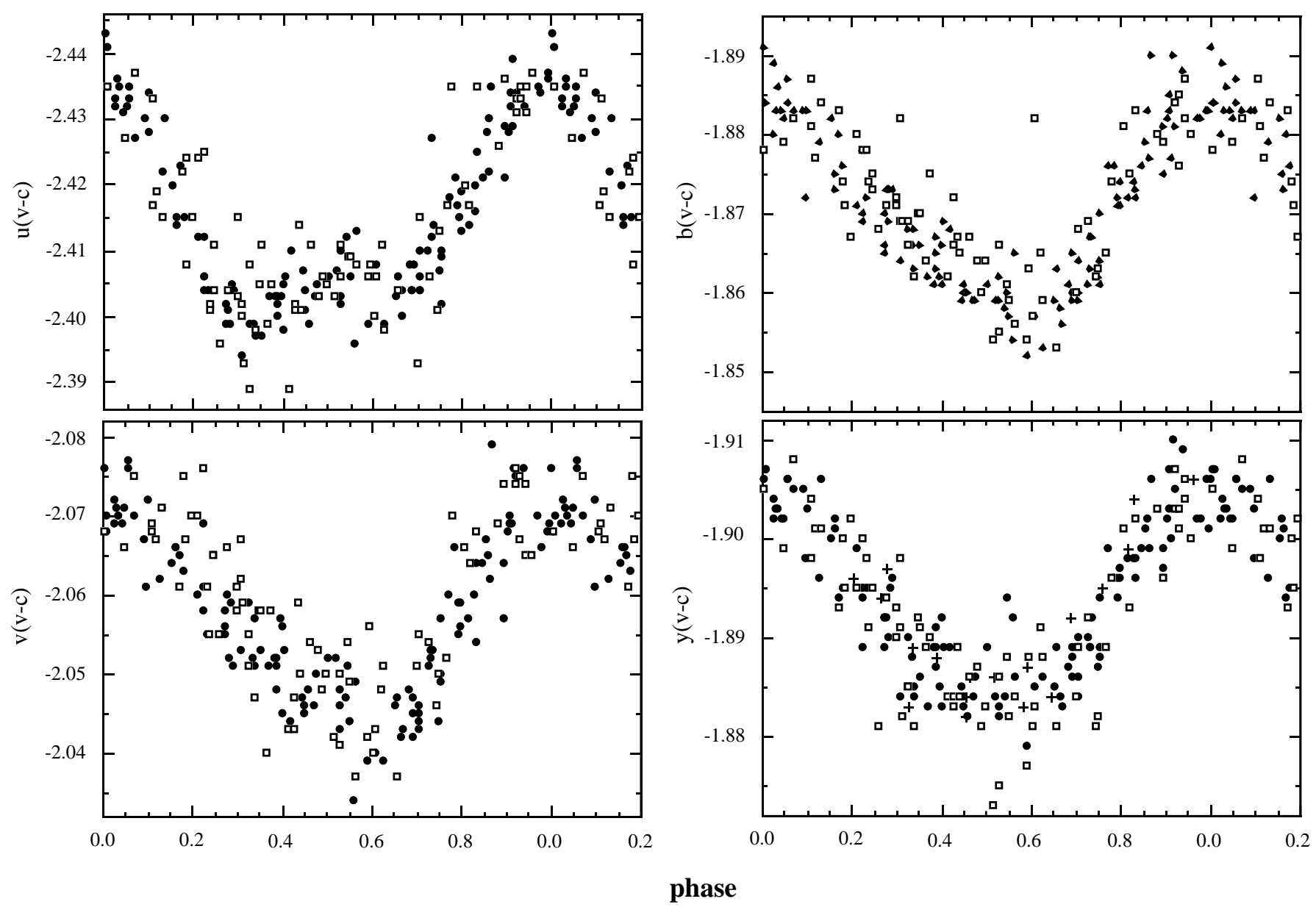

Fig. 3. Differential FCAPT uvby photometry of HR 1297 plotted with the ephemeris $\operatorname{HJD}\left(y_{\max }\right)=2441247.475+15.7490 E$. The FCAPT values of this paper are shown as open squares and those of Adelman \& Brunhouse (1998) as solid circles. In the $y$ graph, the $V$ values of Winzer (1974) plotted with the $y$ zero point of Adelman \& Brunhouse are shown as + signs

\section{4. $12 \mathrm{CMa}$}

Pedersen \& Thomsen (1977) first found the spectrum variability of 12 CMa (HR 2509, HD 49333), a He weak star which is slightly hotter than the Si stars. According to Levato et al. (1996) its $v \sin i=60 \mathrm{~km} \mathrm{~s}^{-1}$. Bohlender et al. (1993) using their own magnetic measurements of this star and previous measurements of the strength of He I $\lambda 4026$ derived an ephemeris

$$
\mathrm{JD}\left(\max B_{1}\right)=2447311.565+(2.18010 \pm 0.00020) E .
$$

For both the 1997-98 and the 1999-2000 observing seasons 33 sets of uvby data were obtained. When the Scargle (1982) algorithm was used a period of 2.17969 days was indicated. However, the 2.18010 day period was found to give better agreement between the two years' worth of data especially for the primary minimum and so the above ephemeris was adopted.

Figure 4 shows the light curves. The light minima are centered near phase 0.25 which is He I line strength maximum and where the magnetic equator crosses the line of sight. The other phase of $B_{1}=0$ occurs at phase 0.75 . The light curves are in phase with amplitudes of about $0.065 \mathrm{mag}, 0.045 \mathrm{mag}, 0.040 \mathrm{mag}$, and $0.035 \mathrm{mag}$ for $u, v$, $b$, and $y$, respectively. The primary minimum is definitely asymmetric.

\section{HD 134214}

HD 134214 (BD $\left.-13^{\circ} 4081\right)$ is the rapidly oscillating Ap stars with the highest principal oscillation frequency at about $2949 \mu \mathrm{Hz}$. It shows a peak-to-peak variation in its pulsational frequency on a time scale of approximately 248 days with a semi-amplitude in $B$ about 3.5 mmag (Kreidl et al. 1994). Their data shows no evidence of amplitude modulation. During the 1997-98, 1998-99, and 1999-2000 observing seasons 29,33 , and 32 sets of uvby photometry, respectively, were obtained with the FCAPT. There is no evidence of periodicity when the data is examined by the Scargle (1982) periodogram. Usually only one observation per night was obtained, but for a few nights two observations per night were made. But only with closely spaced 


\section{CMa}
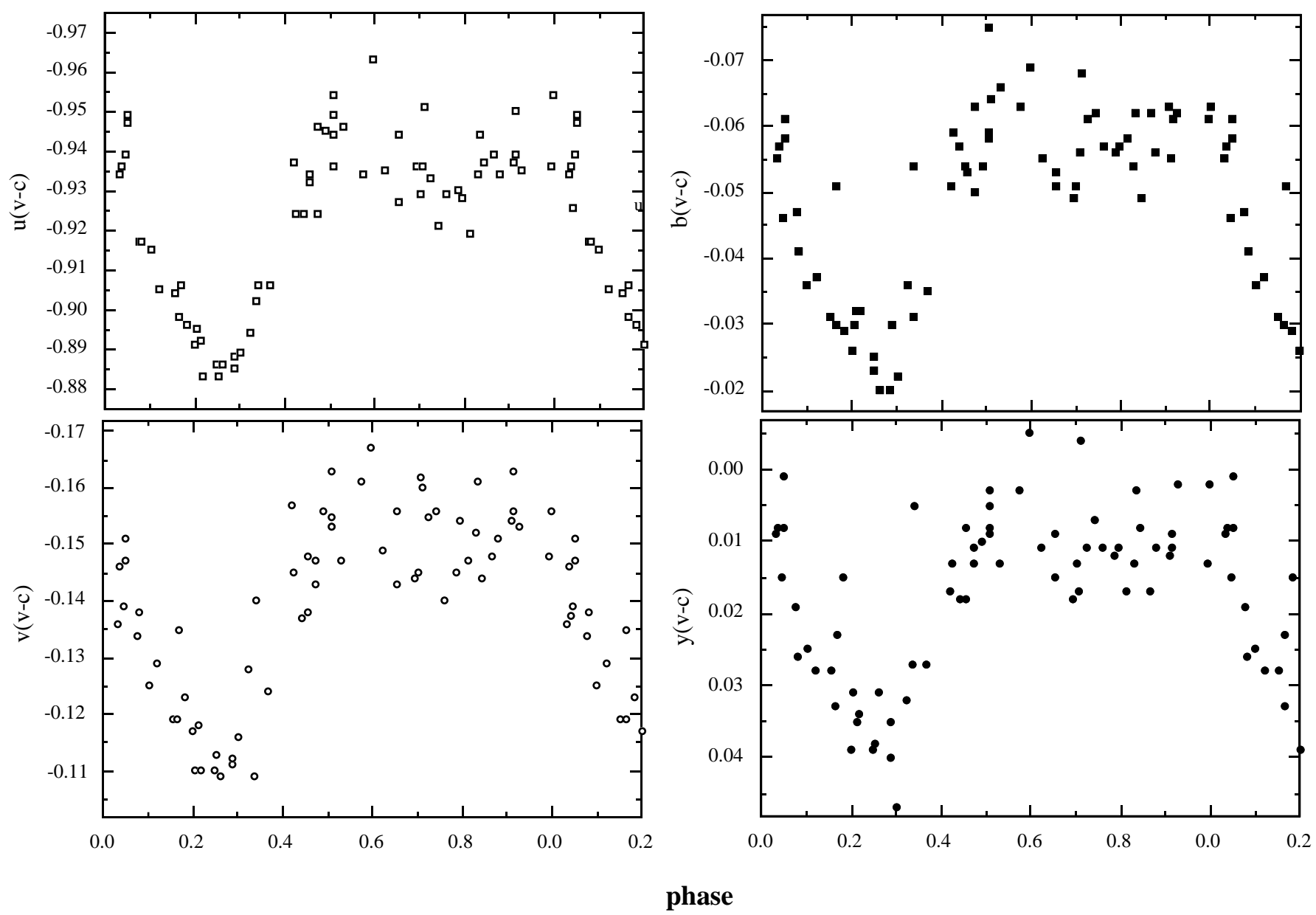

Fig. 4. Differential FCAPT uvby photometry of 12 CMa using the ephemeris $\operatorname{JD}\left(\max B_{1}\right)=2447311.565+2.18010 E$. Each filter is plotted using a different symbol

continuous observations will one find the principal oscillation frequency. The standard deviations for the difference of the check-comparison stars (Table 5) are slightly larger than that for other pairs in this series of papers, but these stars are observed through a greater airmass than most other stars.

Acknowledgements. This work was supported in part by NSF grant AST-9528506. I appreciate the continuing efforts of Louis J. Boyd, Robert J. Dukes Jr., and George P. McCook to keep the FCAPT operating properly. This research has made use of the SIMBAD database, operated at the CDS, Strasbourg, France.

\section{References}

Adelman S.J., Boyce P.W., 1995, A\&AS 114, 253

Adelman S.J., Brunhouse E.F., 1998, PASP 110, 1304

Adelman S.J., Pi C.-L.M., Rayle K.E., 1998, A\&AS 133, 197

Adelman S.J., Rayle K.E., Pi C.-L.M., 1999, A\&AS 136, 379
Bohlender D.A., Landstreet J.D., Thompson I.B., 1993, A\&A 269,355

Catanzaro G., Leone F., Catalano F.A., 1999, A\&AS 134, 211

ESA, 1997, The Hipparcos and Tycho Catalogs, SP-1200

Genet R.M., Boyd L.J., Hayes D.S., et al., 1987, Comm. IAPPP 29,42

Hoffleit D., 1982, The Bright Star Catalogue, 4th edition. Yale University Observatory, New Haven, CT

Horne J.H., Baliunas S.L., 1986, ApJ 302, 757

Kreidl T.J., Kurtz D.W., Schneider H., van Wyk F., et al., 1994, MNRAS 270, 115

Levato H., Malaroda S., Morrell N., Solivella G., Grosso M., 1996, A\&AS 118, 481

Michaud G., Proffitt C.R., 1993, in ASP Conf. Proc. 44, Peculiar Versus Normal Phenomena in A-Type and Related Stars, Dworetsky M.M., Castelli F., Faraggiana R. (eds.). ASP, San Francisco, p. 439

Pedersen H., Thomsen B., 1977, A\&AS 30, 11

Renson P., Manfroid J., 1981, A\&AS 44, 23

Roberts D.H., Lehar J., Dreher J.W., 1987, AJ 93, 968

Scargle J.D., 1982, ApJ 263, 835

Winzer J.E., 1974, Ph.D. Thesis, University of Toronto 